\title{
Damage to Olfactory Progenitor Cells Is Involved in Cigarette Smoke-Induced Olfactory Dysfunction in Mice
}

\author{
Rumi Ueha, ${ }^{*}$ Satoshi Ueha, ${ }^{\dagger}$ Kenji Kondo, ${ }^{*}$ Takashi Sakamoto, ${ }^{*}$ Shu Kikuta, ${ }^{*}$ Kaori Kanaya, ${ }^{\ddagger}$ Hironobu Nishijima, ${ }^{*}$ \\ Kouji Matsushima, ${ }^{\dagger}$ and Tatsuya Yamasoba*
}

\author{
From the Departments of Otolaryngology* and Molecular Preventive Medicine, ${ }^{\dagger}$ Graduate School of Medicine, The University of Tokyo, Tokyo; and the \\ Department of Otolaryngology, ${ }^{\ddagger}$ Tokyo Teishin Hospital, Tokyo, Japan
}

\author{
Accepted for publication \\ November 2, 2015 \\ Address correspondence to \\ Rumi Ueha, M.D., Department \\ of Otolaryngology, University of \\ Tokyo, 7-3-1 Hongo Bunkyo- \\ ku, Tokyo 113-8655, Japan. \\ E-mail: ruu1025@yahoo.co.jp.
}

\begin{abstract}
Exposure to cigarette smoke is a major cause of olfactory dysfunction. However, the underlying mechanisms by which cigarette smoke interferes with the highly regenerative olfactory nerve system remain unclear. To investigate whether cigarette smoke induces olfactory dysfunction by disrupting cell proliferation and cell survival in the olfactory epithelium $(\mathrm{OE})$, we developed a mouse model of smoking that involved intranasal administration of a cigarette smoke solution (CSS). Immunohistological analyses and behavioral testing showed that CSS administration during a period of 24 days reduced the number of olfactory marker protein-positive mature olfactory receptor neurons (ORNs) in the OE and induced olfactory dysfunction. These changes coincided with a reduction in the number of SOX2 ${ }^{+}$ORN progenitors and $\mathrm{Ki}-67^{+}$proliferating cells in the basal layer of the $0 \mathrm{E}$, an increase in the number of caspase $-3^{+}$apoptotic cells, and an increase in the expression of mRNA for the inflammatory cytokines IL-1 $\beta$ and IL-6. Notably, the proliferating ORN progenitor population recovered after cessation of treatment with CSS, resulting in the subsequent restoration of mature ORN numbers and olfaction. These results suggest that SOX2 ${ }^{+}$ORN progenitors are targets of CSS-induced impairment of the $0 \mathrm{E}$, and that by damaging the ORN progenitor population and increasing ORN death, CSS exposure eventually overwhelms the regenerative capacity of the epithelium, resulting in reduced numbers of mature ORNs and olfactory dysfunction. (Am J Pathol 2016, 186: 579-586; http://dx.doi.org/10.1016/ j.ajpath.2015.11.009)
\end{abstract}

Cigarette smoke represents a major source of exposure to toxic chemicals for humans and causes a diverse range of preventable illnesses. ${ }^{1,2}$ The numerous chemical irritants contained in cigarette smoke trigger the generation of reactive oxygen and nitrogen species and expression of inflammatory mediators, such as IL-1 $\beta$, IL-6, and tumor necrosis factor, in the respiratory tract. ${ }^{3}$ Because these mediators damage epithelial tissue and induce inflammatory responses in the respiratory tract, a long history of cigarette smoking significantly increases the risk of various pulmonary diseases, such as chronic obstructive pulmonary disease and lung cancer. ${ }^{3,4}$ In addition to lower respiratory diseases, cigarette smoke causes upper respiratory disorders, such as nasal polyps ${ }^{5}$ and chronic rhinosinusitis, and also impairs olfaction (hyposmia/anosmia). ${ }^{6-8}$ However, the cellular and molecular mechanisms by which cigarette smoke disrupts olfaction remain largely unclear.

Olfaction is mediated by the olfactory system, which is composed of olfactory receptor neurons (ORNs) in the nasal cavity and the olfactory bulb in the forebrain. ${ }^{9}$ Olfactory dysfunction is associated with damage to ORNs and/or the olfactory bulb, which can occur because of a variety of causes, such as exposure to toxic chemicals, airway allergy, upper-airway viral infections, head trauma, and neurodegenerative diseases. ${ }^{10,11}$ Cigarette smoke is a major cause of

Supported by Japan Society for the Promotion of Science grant-in-aid for Scientific Research 24791749 and the Smoking Research Foundation (Tokyo, Japan).

Disclosures: None declared. 
hyposmia and anosmia. ${ }^{6-8}$ Cigarette smoke decreases the thickness of the olfactory epithelium $(\mathrm{OE})^{12}$ and increases apoptosis in the OE. ${ }^{11}$ However, ORNs have regenerative potential through the olfactory epithelial stem cell system, ${ }^{13}$ and thus the extent to which apoptosis causes olfactory dysfunction remains unclear.

Two types of cells in the basal layer of the OE, globose basal cells and horizontal basal cells, act as ORN stem and progenitor cells and produce mature ORNs expressing olfactory marker protein (OMP), which is expressed exclusively in mature ORNs and modulates olfactory signal transduction. ${ }^{14}$ Recently, it was suggested that globose basal cells are actively proliferating multipotent progenitor cells that display increased proliferation after OE damage, ${ }^{15}$ whereas horizontal basal cells are quiescent stem cells that contribute to $\mathrm{OE}$ regeneration only after severe $\mathrm{OE}$ damage. $^{16,17}$ SOX2 is a transcription factor that is widely expressed in stem cell populations, including in neural stem cells, and that plays a role in maintaining cells in an undifferentiated state. ${ }^{18}$ In the OE, SOX2 is expressed by progenitor cells and regulates homeostasis. ${ }^{10,13,19-21}$ Accumulating evidence suggests that SOX2 expression is suppressed by inflammatory cytokines, such as IL-6, resulting in a loss of stemness in multipotent cells. ${ }^{22}$ In addition, we recently demonstrated that $\mathrm{SOX}^{+}$ORN progenitors are damaged by viral infection, alone or in combination with airway allergy, leading to the eventual loss of ORNs. ${ }^{10} \mathrm{We}$, therefore, hypothesized that cigarette smoke-induced inflammation might also disrupt the olfactory progenitor cell system.

Herein, we explored the effects of cigarette smoke on ORNs and ORN progenitors using a newly established mouse model of smoking that involved administration of a cigarette smoke solution (CSS). We also investigated the extent to which CSS-induced damage to the ORNs recovers after cessation of exposure to CSS. Using histological analyses, olfactory habituation/dishabituation tests, and quantitative RT-PCR (RT-qPCR) analyses, we found that cigarette smoke impairs not only $\mathrm{OMP}^{+}$mature ORNs, but also $\mathrm{SOX}^{+}$ORN progenitors. Decreased numbers of ORNs and their progenitors were associated with olfactory disorder that gradually recovered as inflammatory cytokine levels decreased after cessation of exposure to CSS.

\section{Materials and Methods}

Mice

Eight-week-old male C57BL/6 mice were purchased from Saitama Experimental Animals (Saitama, Japan). Mice were housed in a temperature-controlled environment under a 12hour light-dark cycle with access to food and water ad libitum. All animal experiments were conducted in accordance with institutional guidelines and with the approval of the Animal Care and Use Committee of the University of Tokyo (approval number P14-086; Tokyo, Japan).

\section{Mouse Model of Smoking}

The mouse model of cigarette smoking was adapted from a guinea pig model that used CSS. ${ }^{23}$ CSS, prepared by bubbling a stream of the smoke of $40 \mathrm{Hi}$-Lite cigarettes (Japan Tobacco Inc., Tokyo) through $40 \mathrm{~mL}$ of saline, was purchased from CMIC Bioresearch Center Co, Ltd (Tokyo, Japan). We defined the day of final CSS administration as day 0 . CSS $(20 \mu \mathrm{L}$ per animal per time) was administered intranasally once a day on days -23 to -19 . This cycle of four consecutive CSS administrations, followed by 1 rest day, was then repeated, with cycles 2 to 5 conducted over days -18 to $-14,-13$ to $-9,-8$ to -4 , and -3 to 0 , respectively (Figure 1). Control mice received saline intranasally, according to the same schedule as CSS mice. CSS mice were sacrificed on days $1,7,14$, and 28 after the final administration of CSS. Control mice were sacrificed on day 1 after the final administration of saline.

\section{Tissue Preparation}

Septal nasal mucosa was harvested on days $1,7,14$, and 28 after the final intranasal administration of CSS for histological and RT-qPCR analyses. Immediately after sacrificing the mice, the nasal cavities were gently irrigated with $4 \%$ paraformaldehyde to minimize mechanical damage to the OE. The mandibles were discarded, and trimmed heads were skinned, fixed in $4 \%$ paraformaldehyde for a further 24 hours, and then decalcified for 7 days using Decalcifying Solution B (Wako Pure Chemical Industries, Ltd, Osaka, Japan). After decalcification, the tissues were dehydrated in a series of graded ethanol solutions, followed by embedding in paraffin.

\section{Histological Analysis}

All samples were cut at the level of the anterior end of the olfactory bulb, as described previously. ${ }^{10}$ Paraffin sections ( $4 \mu \mathrm{m}$ thick) were deparaffinized in xylene and rehydrated in ethanol before hematoxylin and eosin staining or immunostaining. Hematoxylin and eosin staining was used for evaluation of whole tissue structure. For immunostaining, deparaffinized sections were treated with $3 \%$ hydrogen

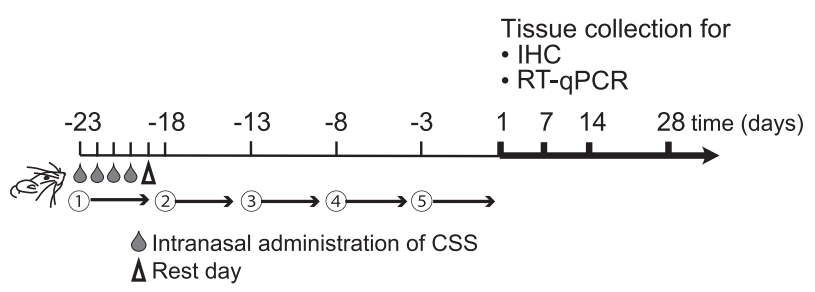

Figure 1 Experimental timeline. Mice were administered intranasally with cigarette smoke solution (CSS) between day -23 and 0 (five cycles, each including four daily doses of $20 \mu \mathrm{L}$ per mouse and 1 rest day). Subsequently, the olfactory epithelium was collected for immunohistochemistry (IHC) and quantitative RT-PCR (qPCR) on the days indicated. 
peroxide to block endogenous peroxidase activity and incubated in Blocking One (Nacalai Tesque, Kyoto, Japan) to block non-specific immunoglobulin binding. Sections were then incubated with primary antibodies against mouse SOX2 (1:300 dilution; rabbit monoclonal; Abcam, Tokyo, Japan; clone EPR3131), Ki-67 (1:200 dilution; rabbit monoclonal; Novus Biologicals, Littleton, $\mathrm{CO}$; number NB600-1252), OMP (1:8000 dilution; goat polyclonal; Wako, Tokyo, Japan; number 544-10001), cleaved capase-3 (1:300 dilution; rabbit polyclonal; Cell Signaling, Tokyo, Japan; number 9661), appropriate peroxidase-conjugated secondary antibodies, and a diaminobenzidine substrate. Analysis of histological sections was restricted to the $\mathrm{OE}$ to minimize variation between specimens. Three different microscopic fields (dorsal, middle, and ventral) of each bilateral septal olfactory neuroepithelium were captured using a digital microscope camera (BZ-9000; Keyence, Osaka, Japan) with a $40 \times$ objective lens. The number of $\mathrm{OMP}^{+}$ORNs was quantified by averaging the number of cells in each of the three microscopic fields between sections. The number of $\mathrm{SOX}^{+}$ORN progenitors, $\mathrm{Ki}-67^{+}$ cells, and caspase- $3^{+}$apoptotic cells per $\mathrm{mm}$ of basal layer length were counted manually for each of the three different fields using Photoshop CS6 digital imaging software version 6.1 (Adobe Systems, San Jose, CA).

\section{RT-qPCR}

Total RNA was isolated from the septal nasal mucosa using TRIzol reagent (Life Technologies, Tokyo, Japan) on days 1, 7, 14, and 28 after the final intranasal administration of CSS, then converted to cDNA using ReverTra Ace qPCR RT Master Mix with gDNA Remover (Toyobo, Tokyo, Japan), according to the manufacturer's instructions. qPCR analysis was performed using THUNDERBIRD Probe qPCR Mix or THUNDERBIRD SYBR qPCR Mix (Toyobo) and an ABI 7500 sequence detector system (Life Technologies). The gene-specific primers and probes used were as follows: Rps3 as endogenous control (Life Technologies assay number Mm00656272_m1); Illb (forward, 5'-AGGCAGGCAGTATCACTCATTGT-3'; reverse, 5'-CGTCACACACCAGCAGGTTATC-3'); and $I l 6$ (forward, 5'-CTGCAAGAGACTTCCATCCAGTT-3'; reverse, 5'-AGGTCTGTTGGGAGTGGTATCC- $3^{\prime}$ ). The expression levels of each gene were normalized to the level of Rps3 expression for each sample.

\section{Behavioral Testing to Evaluate Olfactory Function}

Olfactory sensitivity was evaluated by an olfactory habituation/dishabituation test ${ }^{24}$ that was performed on days 1,7 , and 14 after the final intranasal administration of CSS. Mice were allowed to acclimatize in a clean plastic cage $(46 \times 23.5 \times 20 \mathrm{~cm})$ without wood chip bedding for 30 minutes, then presented with a piece of filter paper soaked in odorless mineral oil (in a 35-mm polystyrene dish) for 3 minutes. This procedure was repeated a total of four times at 1-minute intervals. However, for the fourth exposure, the filter paper was soaked in propyl propionate instead of mineral oil. Throughout each test, mouse behavior was recorded using a digital video camera. The nose of the mouse being within $1 \mathrm{~mm}$ of the filter paper was considered to represent investigative behavior. The duration of investigative behavior was compared between the third and fourth exposures. Mice with normal olfaction display gradually reduced durations of investigative behavior from the first to third exposures (habituation), but display reinstatement of investigative behavior when an odor is presented with the filter paper. A lack of reinstatement indicates reduced or absent olfactory sensitivity.

\section{Statistical Analysis}

Statistical comparisons between groups or time points were performed by paired $t$-tests, the $U$ test, or one-way analysis of variance using GraphPad Prism software version 6.0 (GraphPad Software Inc., San Diego, CA). qPCR data were subjected to logarithmic transformation before analysis. $P<0.05$ was considered to be statistically significant.

\section{Results}

\section{CSS Exposure Impairs ORNs}

We first examined the effect of continuous intranasal administration of CSS on ORNs (Figure 1). Immunohistochemical staining revealed that $\mathrm{OMP}^{+}$mature ORNs formed thick layers in the $\mathrm{OE}$ of saline-treated mice (Figure 2A). In contrast, the number of $\mathrm{OMP}^{+}$cells in the mice that received 20 doses (five cycles) of CSS administration was approximately $70 \%$ less than the numbers observed in saline-treated mice 1 day after the final treatment (Figure 2, A and B). In mice that received 10 doses (2.5 cycles) of CSS administration, no reduction in the number of $\mathrm{OMP}^{+}$mature ORNs was observed. We next examined whether the $\mathrm{OMP}^{+}$mature ORN population would recover after cessation of CSS administration. A time course analysis revealed that the reduction in $\mathrm{OMP}^{+}$ORN numbers became more severe on day 7 after the final intranasal administration of CSS, but then gradually recovered during days 14 to 28 (Figure 2, C and D). This delayed recovery suggests that CSS-induced damage to the $\mathrm{OE}$ is not simply because of acute damage to mature ORNs, but rather that additional long-term factors are involved in impairment of ORNs by CSS.

\section{CSS Exposure Induces Olfactory Dysfunction}

We next examined whether the CSS-induced reduction in $\mathrm{OMP}^{+}$mature ORN numbers is associated with impaired olfaction by using a habituation/dishabituation test to evaluate olfactory sensitivity. In this test, propyl propionate, 
A

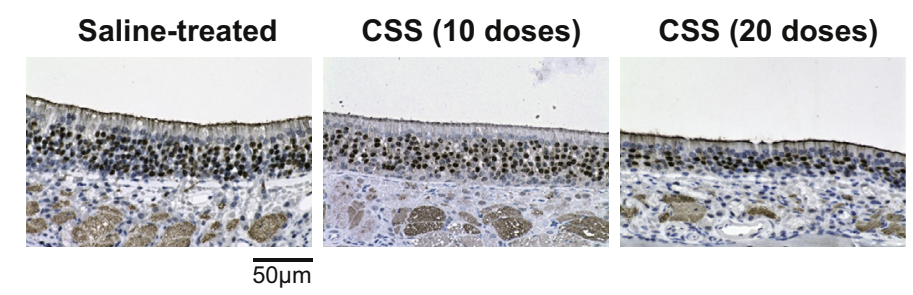

C

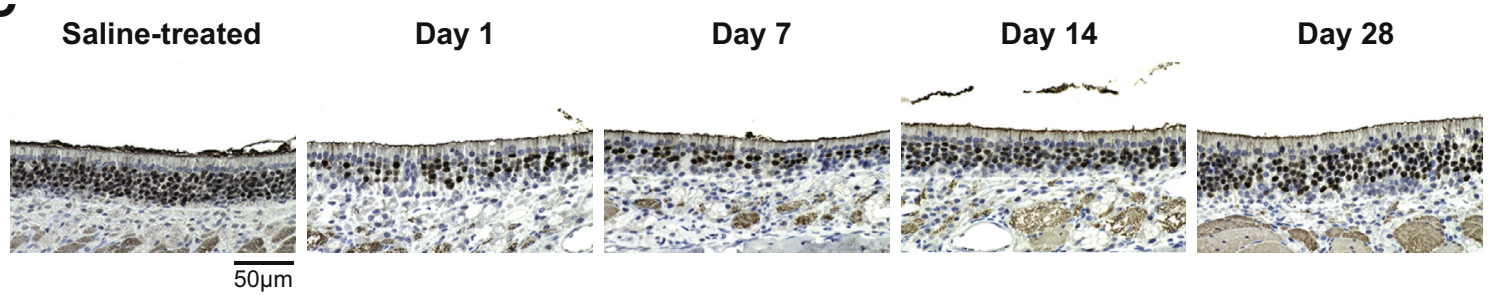

D

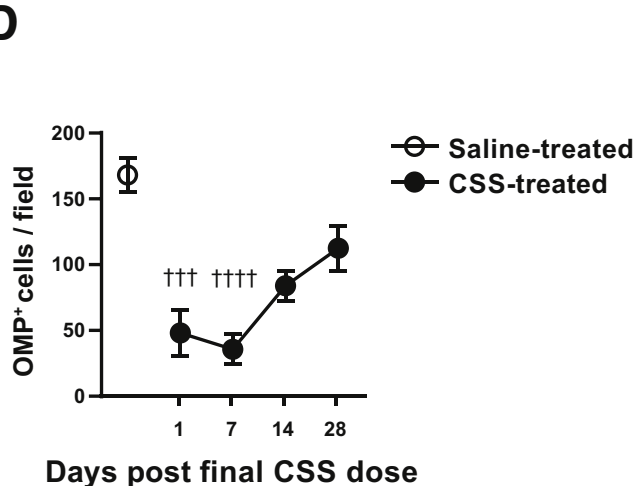

Days post final CSS dose
B

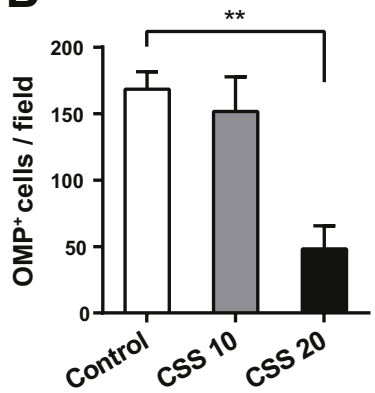

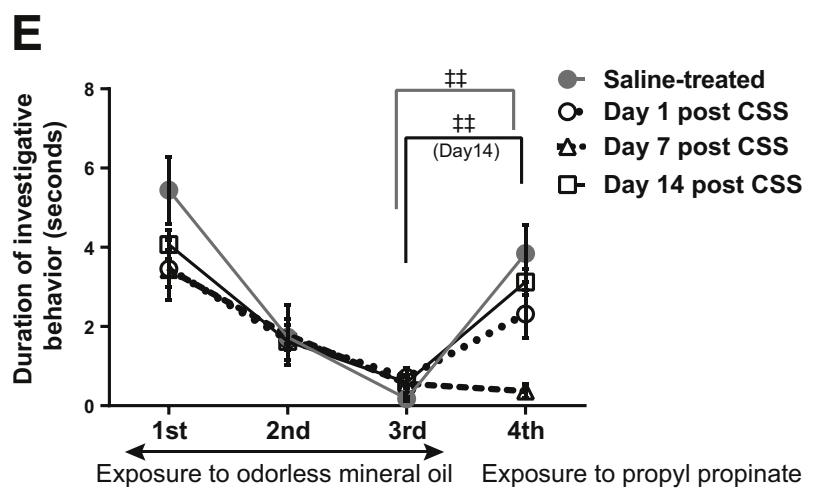

Figure 2 A and B: Immunohistochemical staining (brown) of olfactory marker protein (OMP) ${ }^{+}$cells in the olfactory epithelium 1 day after the final dose of cigarette smoke solution (CSS). Mice received either 10 doses (2.5 cycles) or 20 doses (5 cycles) of CSS. C: Immunohistochemical staining of $\mathrm{OMP}^{+}$cells in the olfactory epithelium in saline-treated mice and on various days after the final CSS dose. Representative images from two independent experiments. D: Number of $\mathrm{MMP}^{+}$olfactory receptor neurons per field in saline- or CSS-treated mice. Data are pooled from two independent experiments. E: Olfactory habituation/dishabituation test. Mice were presented with a piece of filter paper soaked in odorless mineral oil three times for 3 minutes, at 1-minute intervals. For the fourth exposure, the filter paper was soaked in the odorant propyl propionate instead of mineral oil. Data are representative of two independent experiments. No significant differences were detected between exposures 3 and 4 for CSS-treated mice on day $1(P=0.07)$ or day $7(P=0.53)$, suggesting a decrease in olfactory sensitivity. Data represent means \pm SEM $(\mathbf{A}, \mathbf{B}, \mathbf{D}$, and $\mathbf{E}) . n=6(\mathbf{A}-\mathbf{D})$; $n=4$ to $7(\mathbf{E}) .{ }^{* *} P<0.01$ (U test); ${ }^{\dagger \dagger \dagger} P<0.001,{ }^{\dagger \dagger \dagger \dagger} P<0.0001$ versus saline-treated mice (one-way analysis of variance); ${ }^{\ddagger \ddagger} P<0.01$, exposure 3 versus exposure 4 (paired $t$-test).

which is predominantly perceived by the $\mathrm{OE},{ }^{24}$ was used as the odorant. Saline-treated mice displayed decreasing durations of investigative behavior when repeatedly exposed to a piece of filter paper soaked with odorless mineral oil, which is consistent with habituation (Figure 2E). On the fourth exposure, when the filter paper was soaked with propyl propionate, the duration of investigative behavior was significantly longer than that on the third trial, suggesting that the mice were capable of smelling the odorant (dishabituation). In contrast, although CSS-treated mice displayed a similar pattern of habituation as saline-treated mice, there was no significant difference in the duration of investigative behavior between the third and fourth trials on days 1 or 7 after the final CSS administration, suggesting a decrease in olfactory sensitivity. The loss of olfactory sensitivity was most severe on day 7 after the final CSS administration, but recovered by day 14 . These results demonstrate that olfactory function is impaired by CSS administration, but that olfactory function recovers by day 14 after the final CSS administration.

\section{CSS Exposure Impairs ORN Progenitor Cells}

The similarity in the timing of the recovery of $\mathrm{OMP}^{+} \mathrm{ORN}$ numbers and olfaction after cessation of CSS administration suggested that damage to the $\mathrm{OMP}^{+}$ORNs may underlie CSS-induced hyposmia and anosmia. Because the number of $\mathrm{OMP}^{+}$mature ORNs is determined by the balance 
between the cell death of ORNs and the de novo generation of ORNs from ORN progenitors, we next investigated the effects of cigarette smoke on the number of ORN progenitors and cell proliferation, and on cell death, by examining SOX2 expression, Ki-67, and cleaved caspase-3. Caspase-3 is a crucial mediator of programed cell death (apoptosis), which is cleaved during activation into an active form by apoptotic signaling and catalyzes the specific cleavage of many key cellular proteins. ${ }^{25}$ Increases in cleaved caspase-3 expression in the OE have been associated with the clinical loss of sense of smell. ${ }^{26}$ The Ki-67 protein is a specific marker of cell proliferation, ${ }^{27}$ and Ki- $67^{+}$cells are detected throughout the depth of the epithelium (mainly in the basal layer of the OE) ${ }^{28}$ In salinetreated mice, a large number of $\mathrm{SOX}^{+}$ORN progenitors and $\mathrm{Ki}-67^{+}$proliferating cells were detected in the basal layer (Figure 3A). Although SOX2 expression was also observed in sustentacular cells in the luminal layer without progenitor activity, these cells were excluded from our quantification of $\mathrm{SOX}^{+}$ORN progenitors. Cleaved caspase- $3^{+}$apoptotic cells were scarcely detected in the OE, but were somewhat more frequent on days 1,7 , and 14 after final CSS administration. The number of $\mathrm{SOX}_{2}^{+} \mathrm{ORN}$ progenitors in the basal layer was approximately $20 \%$ lower than in saline-treated mice on day 1 after final CSS administration, was elevated on day 7 , but had returned to saline-treated mouse levels by day 14 (Figure 3B). The number of $\mathrm{Ki}-67^{+}$proliferating cells in the basal layer changed with a time course similar to that of $\mathrm{SOX}^{+}$ORN progenitors. Given that $\mathrm{OMP}^{+}$ORNs are maintained by the continuous proliferation and subsequent maturation of $\mathrm{SOX}^{+}$ORN progenitors in the steady-state, these results are consistent with reduced proliferation of ORN progenitors in mice exposed to CSS and the CSS-induced death of ORNs having had a considerable impact on ORN numbers and olfaction.

\section{CSS Administration Induces Increases in IL-1 $\beta$ and IL-6 Expression in the $0 \mathrm{E}$}

Finally, because inflammatory cytokines influence the proliferation and differentiation of neural stem and progenitor cells, we examined the possible involvement of inflammatory cytokines in the CSS-induced disruption of the ORN progenitor cell population. Time course analyses revealed that Il1b and Il6 mRNA expression was significantly

A

Days post final CSS dose

Saline-treated

Day 1

Day 7

Day 14

Day 28
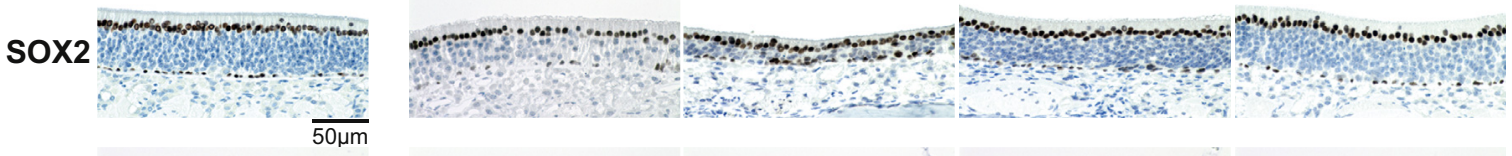

Ki67
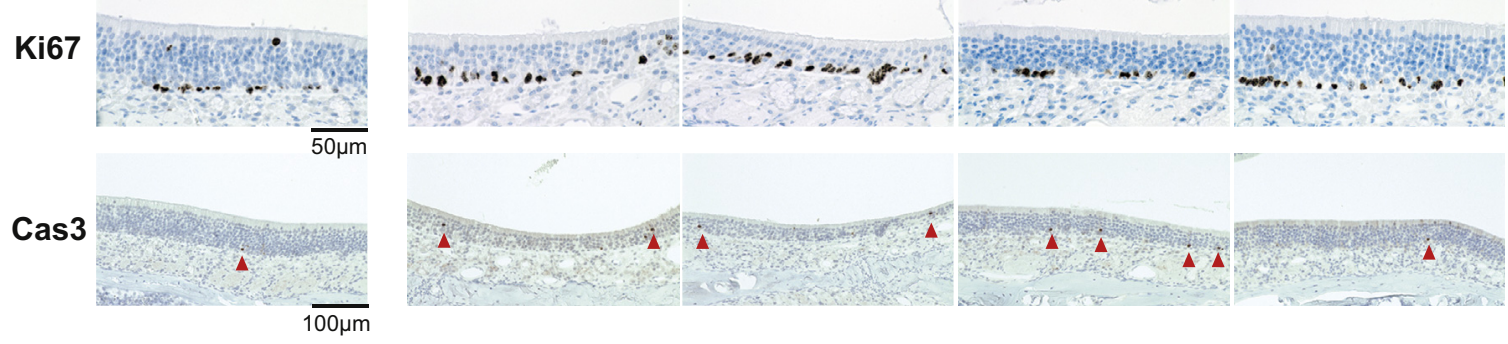

B
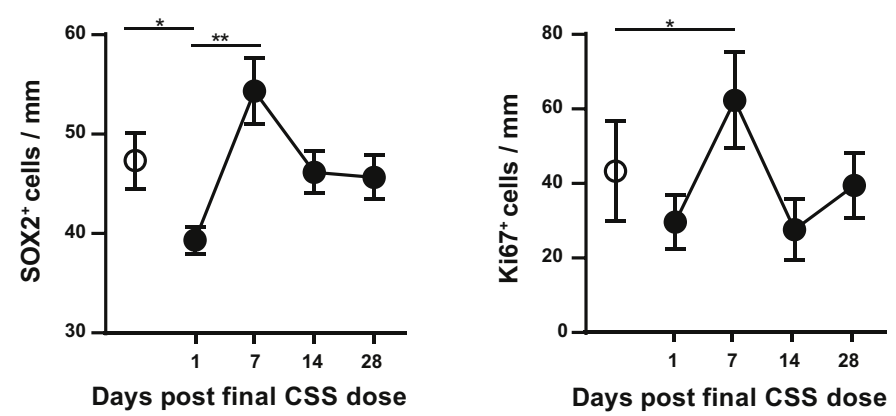

Figure 3 A: Immunohistological staining (brown) of SOX2 ${ }^{+}$olfactory receptor neuron (ORN) progenitor, Ki- $67^{+}$proliferating, and cleaved caspase- $3^{+}$ $\left(\mathrm{Cas}^{+}\right)$apoptotic cells. Tissue sections were counterstained with the nuclear dye hematoxylin (blue). Arrowheads indicate Cas $3^{+}$apoptotic cells in the olfactory epithelium. Representative images from two independent experiments. B: Numbers of SOX2 ${ }^{+}$ORN progenitors and Ki- $67^{+}$actively proliferating cells per $\mathrm{mm}$ of the basal layer in saline (open circles) or cigarette smoke solution (CSS)-treated (closed circles) mice. Data are pooled from two independent experiments. Data represent means \pm SEM (B). $n=6$ (A and B). ${ }^{*} P<0.05,{ }^{* *} P<0.01$ (paired $t$-tests). 

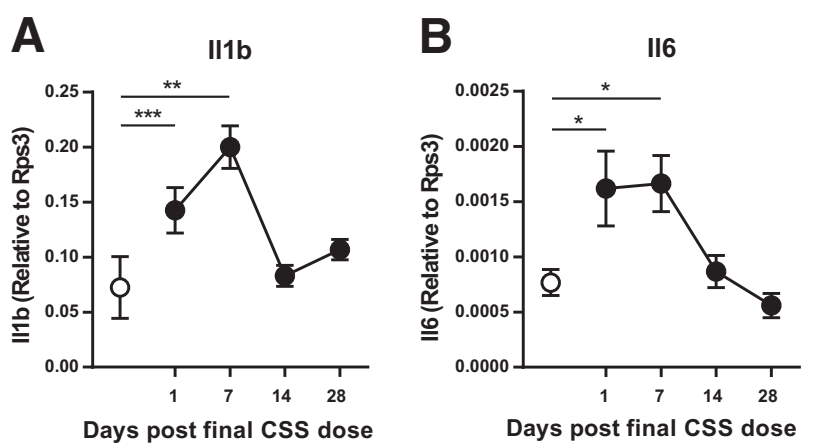

Figure 4 Il1b (A) and Il6 (B) mRNA expression in the nasal mucosa was quantified by quantitative RT-PCR in saline- or cigarette smoke solution (CSS)-treated mice (open and closed circles, respectively), and calculated relative to expression of the endogenous control gene Rps3. Data are representative of two independent experiments. Data represent means \pm SEM. $n=4$ to $6 .{ }^{*} P<0.05,{ }^{*} P P<0.01$, and ${ }^{*} * * P<0.001$ $(U$ test).

elevated in the nasal mucosa on days 1 and 7 after final CSS administration, relative to expression in saline group mice (Figure 4, A and B). However, Illb and Il6 expression returned to the saline-group levels by day 14 after final CSS administration.

\section{Discussion}

Herein, we demonstrate, for the first time, that long-term CSS administration damages not only $\mathrm{OMP}^{+}$mature ORNs but also $\mathrm{SOX}^{+} \mathrm{ORN}$ progenitors in the $\mathrm{OE}$. The reduction in mature ORN numbers was associated with olfactory dysfunction, but cessation of CSS administration resulted in restoration of ORN numbers and olfaction. Time course analyses revealed that the numbers of $\mathrm{SOX}_{2}{ }^{+}$ORN progenitors and $\mathrm{Ki}-67^{+}$proliferating cells increased transiently approximately 7 days after final CSS administration, preceding recovery of the $\mathrm{OMP}^{+}$mature ORN population. Increased apoptosis is unlikely to be solely responsible for the reduction in ORN numbers, because the number of cleaved caspase- $3^{+}$apoptotic cells remained elevated 14 days after final CSS administration, even after the recovery of the $\mathrm{OMP}^{+}$mature ORN population. Elevated expression of the inflammatory cytokines IL- $1 \beta$ and IL- 6 also persisted until day 7 after CSS administration, suggesting that persistent CSS-induced inflammation may influence ORN recovery.

Numerous studies have linked cigarette smoke exposure with olfactory dysfunction in humans, ${ }^{1,2,6-8,29}$ but there are also some studies that report only minimal effects of smoking on olfaction. For example, Yee et $\mathrm{al}^{30}$ observed no adverse impact of smoking history on olfactory function in human patients with chronic rhinosinusitis. Similarly, Rawson et $\mathrm{al}^{31}$ reported a similar proportion of functional olfactory neurons in biopsy specimens from smokers and non-smokers. Differences in the duration and dose of smoking may account for some of these discrepancies.
It has proved challenging to establish an animal model of cigarette smoking that enables investigation of the mechanisms underlying cigarette smoke-induced olfactory dysfunction. In the field of pulmonary research, various experimental models of lung disorders have been described in which guinea pigs, rats, or mice are exposed to cigarette smoke for 3 months or longer. ${ }^{23,32-34}$ However, recently, it was reported that intratracheal CSS administration causes pulmonary impairment in a much shorter time period than exposure to cigarette smoke does. ${ }^{23}$ Herein, we established a practical and reproducible model of cigarette smoking in which olfactory impairment develops after 20 doses of intranasal CSS administration over 24 days. The chemical irritant content of a single dose of CSS administration is approximately equivalent to the exposure a human receives from smoking approximately 40 cigarettes, ${ }^{23}$ and thus our model corresponds to the exposure received by a heavy smoker and is likely to provide insights into the olfactory dysfunction experienced by these smokers. Nevertheless, daily CSS administration does not recapitulate all aspects of cigarette smoking, such as the prolonged period of exposure within each day (time spent smoking) or exposure to heat and ash. Our finding that mice exposed to only 10 doses of CSS did not experience any change in $\mathrm{OMP}^{+}$mature ORN numbers suggests that a certain minimum period of exposure to cigarette smoke is required for the development of olfactory dysfunction. On the other hand, it is possible that longer periods of exposure or higher doses of CSS administration than those used in the present study could cause irreversible $\mathrm{OE}$ impairment and permanent hyposmia/ anosmia.

The OE contains mature ORNs and their progenitors, which are part of the regenerative peripheral nervous system. ${ }^{13}$ The sense of smell is mediated by ORNs that are exposed to the nasal airway, which renders these cells vulnerable to environmental injury and death. As a consequence, a basal level of apoptotic ORN death occurs even in the absence of disease. ${ }^{35}$ Dead ORNs are replaced by the mitosis and maturation of progenitors to maintain sufficient numbers of neurons throughout adult life. Disruption of this balance is proposed to be a common cause for clinical loss of sense of smell. ${ }^{8}$ In our CSS exposure model, we found that a decrease in the numbers of proliferating cells and ORN progenitors and an increase in ORN apoptosis were associated with a reduction in the number of mature ORNs in the OE of CSS-treated mice. The exact mechanism by which cigarette smoke disrupts the balance between proliferation and apoptosis in the OE remains unclear, and there are conflicting reports in the literature on this question. It has been reported that cigarette smoke decreases the thickness of the $\mathrm{OE}$ in humans ${ }^{12}$ and increases apoptosis of the OE in rats. ${ }^{11}$ On the other hand, in a study of patients with chronic rhinosinusitis and a history of smoking, metaplastic hyperplasia was observed, but ORNs and progenitors appeared to persist beneath the epithelium. ${ }^{30}$ Thus, further studies are required to clarify the mechanisms of cigarette 
smoke-induced pathological changes in the OE in rodents and humans. However, it seems likely that cigarette smoke could damage ORNs and their progenitors by inducing inflammation and immune responses in the $\mathrm{OE}$, similar to the way in which cigarette smoke toxicity in the lungs is mediated largely by inflammation and immunotoxicity. Our observation that CSS induces expression of the inflammatory cytokines IL-1 $\beta$ and IL- 6 is consistent with this theory. In particular, IL-6 suppresses the expression of SOX $2,{ }^{22}$ suppresses OE regeneration after injury, and plays an important role in the development of hyposmia. ${ }^{36}$ Considering that elevated levels of IL- $1 \beta$ and IL- 6 expression persisted for at least 7 days after final CSS administration and coincided with the reduced numbers of mature ORNs in the OE, these inflammatory cytokines might be involved in reducing $\mathrm{SOX}^{+}$ORN progenitor numbers, suppressing the maturation of ORNs from their progenitors, or inducing apoptosis. A better understanding of how inflammation affects the ORN progenitor cell system could guide the development of therapeutic strategies for cigarette smoke- or chemical irritant-induced hyposmia and anosmia.

Herein, we focused on the effects of CSS exposure on the OE. However, olfactory dysfunction can also be induced by damage to the olfactory bulb in the forebrain. The ORNs are anatomically linked to olfactory bulbs via the cribriform plate, which enables cigarette smoke and cigarette smokeinduced inflammation to directly impair the central nervous system and affect olfaction. ${ }^{37}$ Accordingly, damage to the olfactory bulb could also be responsible for some olfactory dysfunction. Future neurophysiological studies that quantify signal transduction from the ORNs to the olfactory bulb are necessary to fully understand the pathogenesis of cigarette smoke-induced olfactory dysfunction.

In conclusion, we have demonstrated that by damaging the $\mathrm{SOX}^{+}$ORN progenitor population and increasing ORN death, CSS eventually overwhelms the regenerative capacity of the epithelium, resulting in reduced numbers of mature ORNs and olfactory dysfunction. However, the ORN progenitor population and olfaction recovered after cessation of exposure to CSS. Our findings provide a basis for future studies investigating the mechanisms underlying cigarette smoke-induced damage to ORN progenitor cells, and will help guide the development of preventive and therapeutic approaches for cigarette smoke-induced olfactory dysfunction.

\section{Acknowledgment}

We thank Dr. Francis H.W. Shand for editorial assistance.

\section{References}

1. Swan GE, Lessov-Schlaggar CN: The effects of tobacco smoke and nicotine on cognition and the brain. Neuropsychol Rev 2007, 17:259-273
2. Goncalves RB, Coletta RD, Silverio KG, Benevides L, Casati MZ, da Silva JS, Nociti FH Jr: Impact of smoking on inflammation: overview of molecular mechanisms. Inflamm Res 2011, 60:409-424

3. Nyunoya T, Mebratu Y, Contreras A, Delgado M, Chand HS, Tesfaigzi Y: Molecular processes that drive cigarette smoke-induced epithelial cell fate of the lung. Am J Respir Cell Mol Biol 2014, 50:471-482

4. Hellermann GR, Nagy SB, Kong X, Lockey RF, Mohapatra SS: Mechanism of cigarette smoke condensate-induced acute inflammatory response in human bronchial epithelial cells. Respir Res 2002, 3:22

5. Houser SM, Keen KJ: The role of allergy and smoking in chronic rhinosinusitis and polyposis. Laryngoscope 2008, 118:1521-1527

6. Kushi LH, Byers T, Doyle C, Bandera EV, McCullough M, McTiernan A, Gansler T, Andrews KS, Thun MJ; American Cancer Society Nutrition and Physical Activity Guidelines Advisory Committee: American Cancer Society Guidelines on Nutrition and Physical Activity for cancer prevention: reducing the risk of cancer with healthy food choices and physical activity. CA Cancer J Clin 2006, 56: 254-281; quiz 313-314

7. Chen Y, Dales R, Lin M: The epidemiology of chronic rhinosinusitis in Canadians. Laryngoscope 2003, 113:1199-1205

8. Katotomichelakis M, Balatsouras D, Tripsianis G, Davris S, Maroudias N, Danielides V, Simopoulos C: The effect of smoking on the olfactory function. Rhinology 2007, 45:273-280

9. Su CY, Menuz K, Carlson JR: Olfactory perception: receptors, cells, and circuits. Cell 2009, 139:45-59

10. Ueha R, Mukherjee S, Ueha S, de Almeida Nagata DE, Sakamoto T, Kondo K, Yamasoba T, Lukacs NW, Kunkel SL: Viral disruption of olfactory progenitors is exacerbated in allergic mice. Int Immunopharmacol 2014, 22:242-247

11. Doty RL: The olfactory system and its disorders. Semin Neurol 2009, 29:74-81

12. Kern RC, Conley DB, Haines GK 3rd, Robinson AM: Pathology of the olfactory mucosa: implications for the treatment of olfactory dysfunction. Laryngoscope 2004, 114:279-285

13. Bermingham-McDonogh $\mathrm{O}$, Reh TA: Regulated reprogramming in the regeneration of sensory receptor cells. Neuron 2011, 71:389-405

14. Buiakova OI, Baker H, Scott JW, Farbman A, Kream R, Grillo M, Franzen L, Richman M, Davis LM, Abbondanzo S, Stewart CL, Margolis FL: Olfactory marker protein (OMP) gene deletion causes altered physiological activity of olfactory sensory neurons. Proc Natl Acad Sci U S A 1996, 93:9858-9863

15. Chen X, Fang H, Schwob JE: Multipotency of purified, transplanted globose basal cells in olfactory epithelium. J Comp Neurol 2004, 469: $457-474$

16. Iwai N, Zhou Z, Roop DR, Behringer RR: Horizontal basal cells are multipotent progenitors in normal and injured adult olfactory epithelium. Stem Cells 2008, 26:1298-1306

17. Leung CT, Coulombe PA, Reed RR: Contribution of olfactory neural stem cells to tissue maintenance and regeneration. Nat Neurosci 2007 , 10:720-726

18. Pevny L, Placzek M: SOX genes and neural progenitor identity. Curr Opin Neurobiol 2005, 15:7-13

19. Guo Z, Packard A, Krolewski RC, Harris MT, Manglapus GL, Schwob JE: Expression of pax6 and sox 2 in adult olfactory epithelium. J Comp Neurol 2010, 518:4395-4418

20. Kawauchi S, Kim J, Santos R, Wu HH, Lander AD, Calof AL: Foxg1 promotes olfactory neurogenesis by antagonizing Gdf11. Development 2009, 136:1453-1464

21. Kawauchi S, Shou J, Santos R, Hebert JM, McConnell SK, Mason I, Calof AL: Fgf8 expression defines a morphogenetic center required for olfactory neurogenesis and nasal cavity development in the mouse. Development 2005, 132:5211-5223

22. Yoon DS, Kim YH, Lee S, Lee KM, Park KH, Jang Y, Lee JW: Interleukin-6 induces the lineage commitment of bone marrowderived mesenchymal multipotent cells through down-regulation of Sox 2 by osteogenic transcription factors. FASEB J 2014, 28: $3273-3286$ 
23. Mizutani N, Fuchikami J, Takahashi M, Nabe T, Yoshino S, Kohno S: Pulmonary emphysema induced by cigarette smoke solution and lipopolysaccharide in guinea pigs. Biol Pharm Bull 2009, 32: $1559-1564$

24. Kanaya K, Kondo K, Suzukawa K, Sakamoto T, Kikuta S, Okada K, Yamasoba T: Innate immune responses and neuroepithelial degeneration and regeneration in the mouse olfactory mucosa induced by intranasal administration of Poly(I: C). Cell Tissue Res 2014, 357: 279-299

25. Porter AG, Janicke RU: Emerging roles of caspase-3 in apoptosis. Cell Death Differ 1999, 6:99-104

26. Vent J, Robinson AM, Gentry-Nielsen MJ, Conley DB, Hallworth R, Leopold DA, Kern RC: Pathology of the olfactory epithelium: smoking and ethanol exposure. Laryngoscope 2004, 114:1383-1388

27. Starborg M, Gell K, Brundell E, Hoog C: The murine Ki-67 cell proliferation antigen accumulates in the nucleolar and heterochromatic regions of interphase cells and at the periphery of the mitotic chromosomes in a process essential for cell cycle progression. J Cell Sci 1996, 109(Pt 1):143-153

28. Suzukawa K, Kondo K, Kanaya K, Sakamoto T, Watanabe K, Ushio M, Kaga K, Yamasoba T: Age-related changes of the regeneration mode in the mouse peripheral olfactory system following olfactotoxic drug methimazole-induced damage. J Comp Neurol 2011, 519: 2154-2174

29. Veyseller B, Ozucer B, Karaaltin AB, Yildirim Y, Degirmenci N, Aksoy F, Ozturan O: Connecticut (CCCRC) Olfactory Test: normative values in 426 healthy volunteers. Indian J Otolaryngol Head Neck Surg 2014, 66:31-34
30. Yee KK, Pribitkin EA, Cowart BJ, Vainius AA, Klock CT, Rosen D, Hahn CG, Rawson NE: Smoking-associated squamous metaplasia in olfactory mucosa of patients with chronic rhinosinusitis. Toxicol Pathol 2009, 37:594-598

31. Rawson NE, Gomez G, Cowart B, Brand JG, Lowry LD, Pribitkin EA, Restrepo D: Selectivity and response characteristics of human olfactory neurons. J Neurophysiol 1997, 77:1606-1613

32. Wright JL, Farmer SG, Churg A: Synthetic serine elastase inhibitor reduces cigarette smoke-induced emphysema in guinea pigs. Am J Respir Crit Care Med 2002, 166:954-960

33. Kang MJ, Homer RJ, Gallo A, Lee CG, Crothers KA, Cho SJ, Rochester C, Cain H, Chupp G, Yoon HJ, Elias JA: IL-18 is induced and IL-18 receptor alpha plays a critical role in the pathogenesis of cigarette smoke-induced pulmonary emphysema and inflammation. J Immunol 2007, 178:1948-1959

34. Maeno T, Houghton AM, Quintero PA, Grumelli S, Owen CA, Shapiro SD: CD8 $+\mathrm{T}$ cells are required for inflammation and destruction in cigarette smoke-induced emphysema in mice. J Immunol 2007, 178:8090-8096

35. Deckner ML, Risling M, Frisen J: Apoptotic death of olfactory sensory neurons in the adult rat. Exp Neurol 1997, 143:132-140

36. Xie F, Fang C, Schnittke N, Schwob JE, Ding X: Mechanisms of permanent loss of olfactory receptor neurons induced by the herbicide 2,6-dichlorobenzonitrile: effects on stem cells and noninvolvement of acute induction of the inflammatory cytokine IL-6. Toxicol Appl Pharmacol 2013, 272:598-607

37. Hoffmann D, Wynder EL: Smoke of cigarettes and little cigars: an analytical comparison. Science 1972, 178:1197-1199 